\title{
DENSITY OF BLOCH WAVES AFTER A QUENCH
}

\author{
Jacek Dziarmaga* \\ Institute of Physics, Jagiellonian University, Reymonta 4, 30-059 Kraków, Poland
}

(September 10, 1998)

\begin{abstract}
Production of Bloch waves during a rapid quench is studied by analytical and numerical methods. The density of Bloch waves decays exponentially with the quench time. It also strongly depends on temperature. Very few textures are produced for temperatures lower than a characteristic temperature proportional to the square of the magnetic field.
\end{abstract}

Topological defects appear in many condensed matter systems [1]. They are also believed to play a role in cosmology [2] and in nuclear physics [3]. Kibble [4] pointed out that the defects can be produced in significant numbers during a rapid second order transition from the disordered to the symmetry broken phase. The speed of light was the key factor in his estimate of the defect density just after a quench. Zurek [5] stressed the importance of the nonequilibrium Ginzburg-Landau dynamics of the order parameter. According to the Kibble-Zurek scenario the density of kinks, vortices or monopoles scales like a certain defect-dependent power of the quench rate. The theory was verified in several experiments [6].

Both theory and experiment concentrated so far on hedgehogs, which have a nontrivial asymptote at spatial infinity. Textures are a wide class of topological defects with a constant asymptote. A texture which arises in a $D+1$ component spin system has $D$ spatial dimensions. As the spin vector is constrained to have definite magnitude, it lays on a $D$ dimensional sphere. An external magnetic field prefers, say, downward spin orientation. A spin field has finite energy if its spins point down at spatial infinity. The $D$ dimensional space is compactified to a $D$ dimensional sphere. A finite energy spin field is a map $S^{D} \rightarrow S^{D}$. If the map has a nonzero winding number, then it can not be continuously deformed to a uniform spin-down ground state.

The simplest example of a topological texture is a Bloch wave in one spatial dimension. Its spin is an inplane arrow of definite length, which points down far from the wave. As we pass through the (anti-)texture from the left to the right the spin makes the full (anti-)clockwise rotation. The Bloch wave has a definite size as a result of balance between the Zeeman and the exchange energy.

In this paper we study the production of Bloch waves during a rapid quench. We believe that our results are qualitatively correct for all topological textures like skyrmions in planar ferromagnetic systems (e.g. quan-

${ }^{*}$ E-mail: ufjacekd@thrisc.if.uj.edu.pl tum Hall ferromagnets [7]), magnetic bubbles or Hopfions in three dimensional ferromagnets [8] and baryons in the Skyrme model [3]. In addition, Bloch waves are formally equivalent to fluxons in long Josephson junctions [9].

The Bloch wave is topologically stable because its spin is constrained to lay on a circle. Above the critical temperature the spin (the order parameter) expectation value is zero. There are no textures there. Some nonzero density of textures can be created during a paramagnet $\rightarrow$ ferromagnet transition. No textures are created in a quasistatic transition because the correlation length diverges at the critical point and the ferromagnetic phase is entered already with a long range order (quasi long range order in one spatial dimension). However, if the transition preceeds at a finite rate, then, according to the Kibble-Zurek scenario, the system passes the critical point out of thermal equilibrium. It enters the ferromagnetic phase with a finite correlation length. This lack of long range order can manifest itself with a finite density of topological textures. We will see that the density of Bloch waves created in this way depends not only on the transition rate but also on the external magnetic field. The leading dependence of the density on the transition rate is exponential, which is much in contrast to the power law dependence characteristic for hedgehogs.

\section{Time Dependent Ginzburg-Landau Model}

Let us define a one dimensional time dependent Ginzburg-Landau model by the stochastic field equations for the two spin components $\phi_{\alpha}(t, x), \alpha=1,2$,

$$
\dot{\phi}_{\alpha}=\phi_{\alpha}^{\prime \prime}-a(t) \phi_{\alpha}-\left[\phi_{\beta} \phi_{\beta}\right] \phi_{\alpha}-B \delta_{\alpha 2}+\eta_{\alpha}
$$

where ${ }^{\cdot} \equiv \partial_{t},{ }^{\prime} \equiv \partial_{x} . \quad \eta_{\alpha}(t, x)$ 's are Gaussian noises of temperature $T$ with correlations

$$
\begin{aligned}
& \left\langle\eta_{\alpha}(t, x)\right\rangle=0 \\
& \left\langle\eta_{\alpha}\left(t_{1}, x_{1}\right) \eta_{\beta}\left(t_{2}, x_{2}\right)\right\rangle=2 T \delta_{\alpha \beta} \delta\left(t_{1}-t_{2}\right) \delta\left(x_{1}-x_{2}\right) .
\end{aligned}
$$

The coefficient $a(t)$ is time dependent. We consider a symmetric linear quench

$$
a(t)= \begin{cases}1 & , \text { if } t \leq 0 \\ 1-\frac{t}{\tau} & , \text { if } 0<t \leq 2 \tau \\ -1 & , \text { if } 2 \tau \leq t\end{cases}
$$

Before the quench, for $t<0$, the system is in a symmetric (paramagnetic) phase $(a>0)$, during the quench, at $t=\tau$, it undergoes a transition from the symmetric phase 
$(a(t<\tau)>0)$ to a broken symmetry (ferromagnetic) phase $(a(t>\tau)<0)$. Finally it settles down at $a(t)=$ -1 .

In the final symmetry broken phase $(a=-1)$ the spin field tends to live at the bottom of the Mexican hat potential $V=-|\vec{\phi}|^{2} / 2+|\vec{\phi}|^{4} / 4$. The bottom of the potential is the circle $|\phi|=1$. One can consider fluctuations in the direction normal to this valley. Their characteristic first relaxation time is $O(1)$. The potential plus the exchange energy generate a length scale $O(1)$ for these normal fluctuations. A nonzero magnetic field $B$ removes degeneracy along the circle. We assume that $B$ is small so that we can approximately write

$$
\phi_{1}+i \phi_{2}=e^{i \chi+i \frac{\pi}{2}}
$$

for the field at the bottom of the potential valley. The spin length is a hard mode so it has a fixed magnitude but its orientation (phase $\chi$ ), which is a soft mode, can vary in space and time. Eqs.(11) with $a=-1$ and $\eta=0$ reduce to the effective equation

$$
\dot{\chi}=\chi^{\prime \prime}-B \sin \chi,
$$

which can be recognized as a diffusive sine-Gordon equation. The length scale in this effective model is $B^{-1 / 2}$ and the second relaxation time is $B^{-1}$. If, as is generic, $B<<1$, then these two scales are much longer than those characteristic for the hard magnitude mode. The model (5) has a static soliton solution

$$
\chi(x)=-4 \arctan \left[\tanh \left(\frac{B^{1 / 2} x}{2}\right)\right] \bmod 2 \pi .
$$

This soliton is just the Bloch wave.

\section{Instantaneous Quench}

Once we distinguished between hard and soft modes with corresponding length and time scales, we can set to the instantaneous quench $(\tau=0$ in Eq.(3i)). In the symmetric phase $(t<\tau)$ the order parameter fluctuates around the ground state $\left(\phi_{1}, \phi_{2}\right)=(0,-B)$. If $B<<1$ and the temperature $T$ is moderate, then the qubic nonlinearity on the RHS. of Eq.(11) can be neglected. We are in the Gaussian regime. The system before the quench has a unit correlation length. As the quench is instantenous, it enters the symmetry broken phase with this correlation length unchanged. Its further evolution can be unambigously divided into two stages.

The first stage is very short, it lasts for $O(1)$ units of time. This is the time the order parameter needs to roll down to the bottom of the sombrero potential. At the end of this stage the spin already has a fixed magnitude, $|\vec{\phi}| \approx 1$. The phase $\chi$ is still chaotic but it is correlated over the length scale $O(1)$. The field already contains Bloch waves but they are very thin $(O(1)$ width) and distorted, see the plot in Fig.1.
In the second long stage, which lasts for another $O\left(B^{-1}\right)$ units of time, the initial "baby Bloch waves" grow in size, according to Eq.(5), until they become fully pfledged Bloch waves (6), compare Fig.1.

We want to estimate the density of the Bloch waves after the quench. Let us first estimate the density but of the baby Bloch waves. We define the position of the (anti)baby Bloch wave by a point $x$ where the spin points up. In the gaussian approximation the field fluctuates around $\left(\phi_{1}, \phi_{2}\right)=(0,-B)$ at $t=0^{-}$. The quadratic potential is turned upside down at $t=0$. The field at $x$ rolls down the slope of the sombrero potential to the upwards spin orientation iff $\bar{\phi}_{2}(x)>B$ and $\bar{\phi}_{1}(x)=0 . \bar{\phi}$ is an average of $\phi$ over the unit correlation length. In our calculations below we take this average by introducing an ultraviolet cut-off in momentum space at $|k|=1$. In the gaussian approximation the probability that the two conditions hold simultaneously is a product of their probabilities. Let us work out the probabilities one by one.

The density of the points such that $\bar{\phi}_{1}(0, x)=0$ can be worked out with the general formula 10

$$
\begin{aligned}
N\left[\bar{\phi}_{1}=0\right] & =\frac{1}{\pi} \sqrt{\frac{\left\langle\bar{\phi}_{1}^{\prime}(0, x) \bar{\phi}_{1}^{\prime}(0, x)\right\rangle}{\left\langle\bar{\phi}_{1}(0, x) \bar{\phi}_{1}(0, x)\right\rangle}}= \\
& =\frac{1}{\pi} \sqrt{\frac{\frac{T}{\pi} \int_{-1}^{+1} d k \frac{k^{2}}{1+k^{2}}}{\frac{T}{\pi} \int_{-1}^{+1} d k \frac{1}{1+k^{2}}}} \approx 0.17 .
\end{aligned}
$$

In the second equality the equilibrium two point correlation function in momentum space at $t=0^{-}$was taken into account.

Now we estimate the probability that $\bar{\phi}_{2}\left(0^{-}, x\right)>B$. At $t=0^{-} \bar{\phi}_{2}$ fluctuates around $-B$. The magnitude of the fluctuation squared can be found as

$$
g^{2}=\left\langle\left[\bar{\phi}_{2}\left(0^{-}, x\right)+B\right]^{2}\right\rangle=\frac{T}{\pi} \int_{-1}^{+1} d k \frac{1}{1+k^{2}}=\frac{T}{2} .
$$

The normalized gaussian probability distribution of $\bar{\phi}_{2}\left(0^{-}, x\right)$ is $p\left(\bar{\phi}_{2}\right)=\exp -\left(\bar{\phi}_{2}+B\right)^{2} / 2 g^{2} / \sqrt{2 \pi g^{2}}$. With this distribution the desired probability is

$$
P\left[\bar{\phi}_{2}>B\right] \equiv \int_{+B}^{+\infty} p(x)=\frac{1}{2}\left\{1-\operatorname{Erf}\left[\frac{2 B}{\sqrt{T}}\right]\right\},
$$

where we use Mathematica's $\operatorname{Erf}[x]=\int_{0}^{x} \frac{2 d y}{\sqrt{\pi}} \exp \left(-y^{2}\right)$. The density of baby Bloch waves scales like

$N_{b a b y}=N\left[\bar{\phi}_{1}=0\right] \times P\left[\bar{\phi}_{2}>B\right]=0.08 c_{1}\left\{1-\operatorname{Erf}\left[c_{2} \frac{2 B}{\sqrt{T}}\right]\right\}$

with extra parameters $c_{1}, c_{2}=O(1)$ to reflect the orderof-magnitude nature of our calculations.

$N_{b a b y}$ does not distinguish between textures and antitextures. Some pairs of baby textures and antitextures 
will annihilate. In the second slow stage of the relaxation process a baby texture grows in width to its full mature size $\approx 3 \times \frac{4}{\sqrt{B}}$. If there is a baby antitexture within a comparable distance, the texture and antitexture annihilate each other. An average topological charge density within this distance is zero. Fluctuation around this average density is the final density of Bloch waves $N$ and it is proportional to $\sqrt{\frac{\sqrt{B}}{12} \times N_{b a b y}}$

$$
N=0.08 C_{1} B^{1 / 4} \sqrt{1-\operatorname{Erf}\left[C_{2} \frac{2 B}{\sqrt{T}}\right]} \text {, if } N<<B^{1 / 2} .
$$

with constants $C_{1}, C_{2}=O(1)$. The formula is accurate in the dilute regime, $N<<B^{1 / 2}$. When extrapolated to the oversaturated regime, it gives an upper estimate. The curve (11) favourably compares with the results of our numerical simulations, see Fig2.

Density of Bloch waves depends in a critical way on temperature. Very few textures are produced for temperatures lower than $B^{2}$. This can be easily understood. If $T<<B^{2}$, then just before the quench the field is localized in a very close neighbourhood of the ground state $\left(\phi_{1}, \phi_{2}\right)=(0,-B)$. After the quadratic potential is reversed, the field uniformly (up to negligible fluctuations) rolls down to $(0,-1)$ without any chance to wind around the top of the Mexican hat potential.

\section{Finite Rate Quench}

We first estimate the density of baby Bloch waves at the end of the quench at $t=2 \tau$. Let us define $m(t)=\left\langle\phi_{2}(t, x)\right\rangle$ as a noise average of $\phi_{2}$ or equivalently $\bar{\phi}_{2}$. A noise average of the linearized $\alpha=2$ component of Eqs.(11) is

$$
\dot{m}(t)=-a(t) m(t)-B
$$

The solution with the initial condition $m(0)=-B$ and $a(t)$ given by (3) at the end of the quench is given by

$$
\begin{gathered}
m(2 \tau)=-B-B \sqrt{2 \pi \tau} e^{\tau / 2} \operatorname{Erf}[\sqrt{\tau / 2}] \\
\tau \gg 1-B \sqrt{2 \pi \tau} e^{\tau / 2} .
\end{gathered}
$$

At the end of the quench the fluctuations of $\bar{\phi}_{2}$ around its average $m(2 \tau)$ are

$$
\begin{aligned}
& g^{2} \equiv\left\langle\left[\bar{\phi}_{2}(2 \tau, x)-m(2 \tau)\right]^{2}\right\rangle=\int_{-1}^{+1} d k G(2 \tau, T, k), \\
& G(2 \tau, T, k)=\frac{T e^{-4 k^{2} \tau}}{2 \pi}\left\{\frac{1}{1+k^{2}}+\sqrt{\pi \tau} e^{\tau\left(1+k^{2}\right)^{2}} \times\right. \\
& \left.\left[\operatorname{Erf}\left(\sqrt{\tau}\left(k^{2}+1\right)\right)-\operatorname{Erf}\left(\sqrt{\tau}\left(k^{2}-1\right)\right)\right]\right\} \stackrel{\tau>>1, k \neq 1}{\approx} \\
& \frac{T}{2} \sqrt{\frac{\tau}{\pi}} e^{\tau\left(1-2 k^{2}\right)}\left[1-\operatorname{Sign}\left(k^{2}-1\right)\right] .
\end{aligned}
$$

For $\tau>>1$ the fluctuations tend to $g^{2} \approx T e^{\tau} / 2 \sqrt{2}$. Similarly as for the instantenous quench the probability that $\bar{\phi}_{2}(2 \tau, x)>B$ is given by

$$
\begin{aligned}
P\left[\bar{\phi}_{2}(2 \tau)>B\right] & =\frac{1}{2}\left\{1-\operatorname{Erf}\left[\frac{B-m(2 \tau)}{\sqrt{2 g^{2}}}\right]\right\} \\
\tau>>\frac{T}{B^{2}}, 1 & \sqrt{\frac{T}{8 \sqrt{2} \pi^{2} \tau B^{2}}} \exp \left\{-\frac{2 \sqrt{2} \pi \tau B^{2}}{T}\right\} .
\end{aligned}
$$

At the end of the quench the density of the points such that $\bar{\phi}_{1}(2 \tau, x)=0$ is given by

$$
\begin{aligned}
N\left[\bar{\phi}_{1}=0\right]= & \frac{1}{\pi} \sqrt{\frac{\left\langle\bar{\phi}_{1}^{\prime}(2 \tau, x) \bar{\phi}_{1}^{\prime}(2 \tau, x)\right\rangle}{\left\langle\bar{\phi}_{1}(2 \tau, x) \bar{\phi}_{1}(2 \tau, x)\right\rangle}}= \\
& \frac{1}{\pi} \sqrt{\frac{\int_{-1}^{+1} d k k^{2} G(2 \tau, T, k)}{\int_{-1}^{+1} d k G(2 \tau, T, k)}} \approx>1 \\
& \frac{1}{2 \pi \sqrt{\tau}} .
\end{aligned}
$$

The density of baby Bloch waves is

$N_{b a b y}\left(\tau>>T / B^{2}\right) \approx \sqrt{\frac{T}{32 \sqrt{2} \pi^{4} \tau B^{2}}} \exp \left\{-\frac{2 \sqrt{2} \pi \tau B^{2}}{T}\right\}$

We obtain the density of Bloch waves in a similar way as for the instantaneous quench,

$$
\begin{aligned}
N\left(\tau>>T / B^{2}\right) & =\sqrt{\frac{\sqrt{B}}{12} \times N_{\text {baby }}}= \\
& =0.035 D_{1}\left(\frac{T}{B \tau}\right)^{1 / 4} \exp \left\{-D_{2} \frac{\sqrt{2} \pi \tau B^{2}}{T}\right\},
\end{aligned}
$$

with constants $D_{1}, D_{2}=O(1)$. The density of textures decays exponentially with $\tau$ on the time scale $O\left(T / B^{2}\right)$. Eq.(18) is compared with numerics in Fig.3.

\section{Conclusion.}

Almost no textures are produced for $T$ less than $B^{2}$. Their density decays exponentially with the quench time. The time scale for this exponential decay is of the order of the first relaxation time.

Our findings have qualitative implications for higher dimensional textures. In the case of Skyrme solitons [3], which model baryons, an effective "magnetic field" is provided by the pion mass term in the Lagrangian. The density of baryons just after a chiral transition should be supressed as compared to the density predicted by the standard Kibble-Zurek scenario. This case deserves a separate quantitative study; the study is given in a preprint 11. 
This work extends the Kibble-Zurek scenario to the case with a bias field which explicitly breaks the $O(D+1)$ symmetry. In the standard scenario domains of characteristic size are formed, each of them has a random 2 orientation chosen with uniform probability distribution from the $D$ dimensional sphere. With a bias field this probability is not uniform. The landscape just after a transition is a uniform sea of spin down with islets of spin up scattered here and there. With an appropriate winding of spin in its neighbourhood an islet can survive as a localized topological texture.

\section{ACKNOWLEDGMENTS}

I would like to thank Leszek Hadasz for help with numerical simulations. This work was supported by the KBN grant 2 P03B 00815.

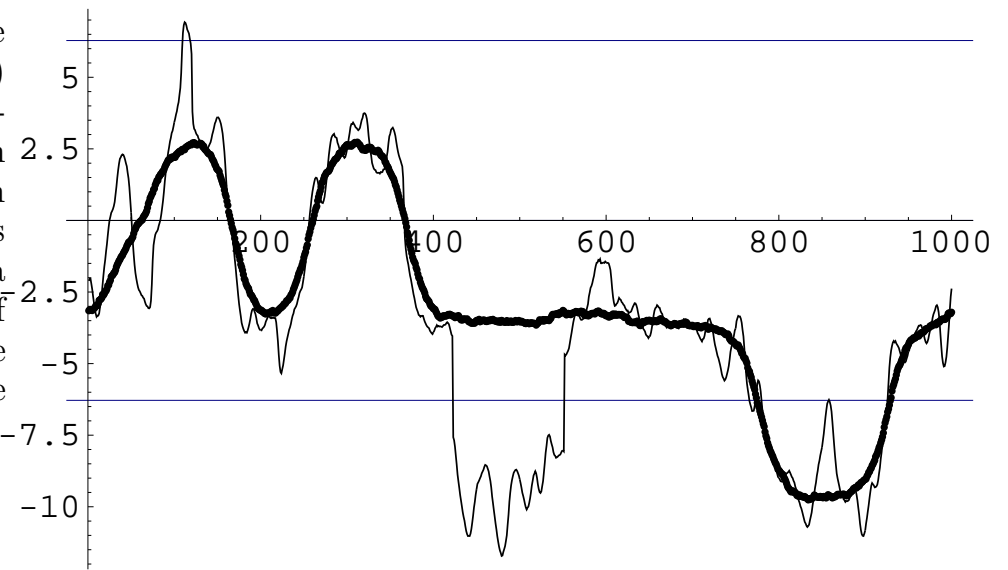

Fig.1. The phase $\chi$ (compare Eq.(何) as a function of $x$ for an instantaneous quench with $B=0.005$ and $T=0.00035$. The thin line is the phase at $t=10$ and the bold line is a plot for $t=1000$. A (baby) Bloch wave can be identified as a place where one of the $-2 \pi, 0,+2 \pi$ gridlines is crossed.

[1] A.S.Davydov,"Solitons in Molecular Systems", Kluwer Academic Publishers, Dordrecht-Boston-London 1990. 0.005

[2] A.Vilenkin and E.P.S.Shellard, "Cosmic Strings and Other Topological Defects", Cambridge Univ. Press, 0.004 Cambridge, 1994.

[3] T.H.R.Skyrme, Proc.Roy.Soc.A260, 127 (1961).

[4] T.W.B.Kibble, J.Phys.A9, 1387 (1976); Phys.Rep.67.0.002= 183 (1980).

[5] W.H.Zurek, Nature 317, 505 (1985); Acta Phys.Polono. 001 B24, 1301 (1993); Phys.Rep.276 (1996) 177.

[6] I.Chuang, R.Dürrer, N.Turok and B.Yurke, Science 251, 1336 (1991); M.J.Bowick, L.Chandar, E.A.Schiff and A.M.Srivastava, Science 263, 943 (1994); P.C.Hendry, N.S.Lawson,

R.A.M.Lee, P.V.E.McClintock and C.H.D.Williams, Nature 368, 315 (1994); V.M.H.Ruutu, V.B.Eltsov, A.J.Gill, T.W.B.Kibble, M.Krusius, Y.G.Makhlin, B.Placais, G.E.Volovik and Wen Xu, Nature 382, 334 (1996); C.Bäuerle, Yu.M.Bunkov, S.N.Fisher, H.Godfrin and G.R.Pickett, Nature 382, 332 (1996).

[7] S.L.Sondhi, A.Karlhede, S.A.Kivelson and E.H.Rezayi, Phys.Rev.B47, 16419 (1993).

[8] V.G.Bar'yakhtar, M.V.Chetkin, B.A.Ivanov and S.N.Gadetskii, "Dynamics of topological magnetic solitons - experiment and theory", Springer Verlag, Berlin, 1994.

[9] A.C.Scott and W.J.Josephson, Appl.Phys.Lett.14, 316 (1969).

[10] B.I.Halperin, "Physics of Defects", proceedings of Les Houches, Session XXXV 1980 NATO ASI (North Holland Press, 1981) p.816; F.Liu and G.F.Mazenko, Phys. Rev. B46, 5963 (1992).

[11] J.Dziarmaga and M.Sadzikowski, hep-ph/9809313. 


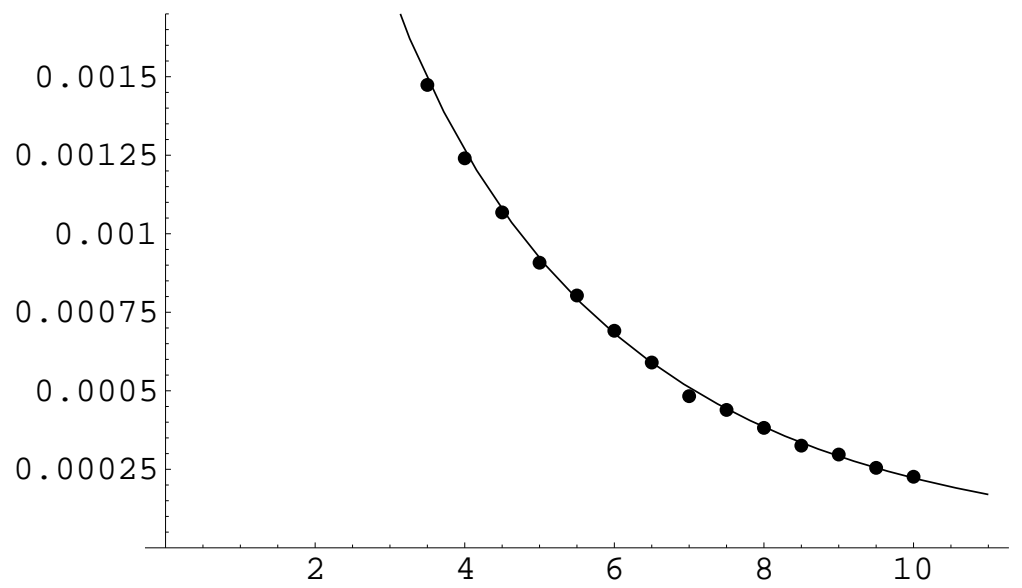

Fig.3. Density of Bloch waves as a function of the quench time $\tau$ for the fixed magnetic field $B=0.005$ and the temperature $T=0.00035$. The solitons were counted at $t=2 \tau+5 / B$. Numerical data were fitted with the curve (18) - solid line. The coefficients are $D_{1}=0.36 \stackrel{+}{-}$ 0.005 and $D_{2}=0.74 \stackrel{+}{-} 0.02$. 